\title{
Turning Thirty, I Contemplate Students Bicycling Home Rita Dove
}

This is the weather of change and clear light. This is weather on its B side, askew, that propels the legs of young men in tight jeans wheeling

through the tired, wise spring. Crickets too awake in choirs out of sight, although I imagine we see the same thing and for a long way.

This, then, weather to start over.

Evening rustles her skirts of sulky organza. Skin prickles, defining what is and shall not be. ...

How private

the complaint of these green hills. 